\title{
The Expanding Role of Contrast-Enhanced Endoscopic Ultrasound in Pancreatobiliary Disease
}

\author{
Jun-Ho Choi* and Dong Wan Seo ${ }^{\dagger}$ \\ ${ }^{*}$ Department of Internal Medicine, Dankook University College of Medicine, Cheonan, and ${ }^{\dagger}$ Division of Gastroenterology, Department of \\ Internal Medicine, Asan Medical Center, University of Ulsan College of Medicine, Seoul, Korea
}

Since its introduction into clinical practice in the 1980s, endoscopic ultrasound (EUS) has been described as a good imaging modality for the diagnosis of pancreatobiliary diseases. However, differential diagnosis of certain lesions based only on B-mode ultrasound images can be challenging. Clinical use of ultrasound contrast agents has expanded the utility of EUS from that of detection to characterization of pancreatobiliary lesions based on the enhancement features of contrastenhanced EUS (CE-EUS). Current low mechanical index techniques for CE-EUS using second-generation contrast agents have a number of distinct advantages over conventional diagnostic modalities in evaluating pancreatobiliary lesions, including real-time assessment of perfusion pattern, availability, and the absence of exposure to radiation. This article describes the technical aspects of CE-EUS and reviews the expanding indications in pancreatobiliary diseases and further development of this technique. (Gut Liver 2015;9:707713)

Key Words: Endosonography; Contrast; Pancreas; Biliary tract

\section{INTRODUCTION}

Endoscopic ultrasound (EUS) has represented a rich source of innovation that has evolved into a technique that has had a substantial clinical impact in digestive diseases. ${ }^{1}$ B-mode ultrasound provides information on structural anatomy, diffuse parenchymal change, as well as focal target lesions. However, depending on the underlying pathology, structural changes become more or less evident.

To overcome the shortcomings of EUS, several techniques of image enhancement have been proposed. The intravenous ap- plication of ultrasound contrast agents (UCAs) is a useful new adjunctive tool in the field of EUS. UCAs consist of gas-filled microbubbles encapsulated by a phospholipid or albumin shell that is injected intravenously, and its uptake-washout pattern through a given lesion is captured by a color or the power Doppler mode of ultrasound. ${ }^{2,3}$ Contrast-enhanced Doppler EUS helps to distinguish between vascular-rich and hypovascular areas. However, contrast-enhanced Doppler EUS suffers from poor spatial resolution as well as blooming and flash artifacts, which can make it difficult to evaluate tumor vasculature. ${ }^{2,3}$ With the advent of harmonic imaging techniques (contrastenhanced harmonic EUS), both parenchymal perfusion and microcirculation can be visualized, resulting in the enhanced characterization of pancreatobiliary diseases. ${ }^{2-4}$ In this paper, we aim to review the technical aspects of contrast-enhanced EUS (CE-EUS), and put them into the appropriate clinical context of pancreatobiliary diseases and future research areas.

\section{ULTRASOUND CONTRAST AGENTS}

Several contrast agents, including Levovist and Albunex (first-generation UCAs), as well as SonoVue, Sonazoid, and Definity (second-generation UCAs), are now commercially available (Table 1). ${ }^{5}$ Second-generation UCAs, containing inert gases with low solubility in water, have been commercially available since 2001. The advantage of second-generation UCAs is that they are able to pass through the lungs, thereby remaining confined inside blood vessel for a longer period of time. In addition, due to low solubility, the second-generation UCAs are more stable, with favorable resonance behavior at low acoustic pressures and thus provide longer specific imaging in a realtime study.

\footnotetext{
Correspondence to: Dong Wan Seo

Division of Gastroenterology, Department of Internal Medicine, Asan Medical Center, University of Ulsan College of Medicine, 88 Olympic-ro 43gil, Songpa-gu, Seoul 05505, Korea

Tel: +82-2-3010-3192, Fax: +82-2-476-0824, E-mail: dwseoamc@amc.seoul.kr Received on February 13, 2015. Accepted on June 25, 2015. pISSN 1976-2283 eISSN 2005-1212 http://dx.doi.org/10.5009/gnl15077

() This is an Open Access article distributed under the terms of the Creative Commons Attribution Non-Commercial License (http://creativecommons.org/licenses/by-nc/4.0) which permits unrestricted non-commercial use, distribution, and reproduction in any medium, provided the original work is properly cited.
} 
Table 1. Ultrasound Contrast Agents ${ }^{5}$

\begin{tabular}{|c|c|c|}
\hline Contrast agent & Composition & Manufacturer \\
\hline \multicolumn{3}{|l|}{ First-generation } \\
\hline Albunex & 5\% Sonicated serum albumin with stabilized microbubbles & Mallinckrodt \\
\hline Echovist & Standardized microbubbles with galactose shell & Schering \\
\hline Levovist & Stabilized, standardized microbubbles with galactose, $0.1 \%$ palmitic acid shell & Schering \\
\hline Myomap & Albumin shell & Quadrant \\
\hline Qantison & Albumin shell & Quadrant \\
\hline Sonavist & Cyanoacrylate shell & Schering \\
\hline \multicolumn{3}{|l|}{ Second-generation } \\
\hline Definity/luminity & $\mathrm{C}_{3} \mathrm{~F}_{8}$ with lipid stabilizer shell & Bristol-Myers Squibb Medical Imaging \\
\hline Sonazoid & $\mathrm{C}_{4} \mathrm{~F}_{10}$ with lipid stabilizer shell & GE Healthcare \\
\hline Imagnet-imavist & $\mathrm{C}_{6} \mathrm{~F}_{14}$ with lipid stabilizer shell & Alliance \\
\hline Optison & $\mathrm{C}_{3} \mathrm{~F}_{8}$ with denatured human albumin shell & GE Healthcare \\
\hline SonoVue & $\mathrm{SF}_{6}$ gas with lipid stabilizer shell & Bracco \\
\hline AI700/imagify & $\mathrm{C}_{4} \mathrm{~F}_{10}$ gas core stabilized with polymer shell & Acusphere \\
\hline \multicolumn{3}{|l|}{ Third-generation } \\
\hline Echogen & Dodecafluoropentane liquid in phase shift colloid emulsion & Sonus Pharmaceuticals \\
\hline
\end{tabular}

\section{SAFETY CONSIDERATIONS}

In general, UCAs are very safe, with a low incidence of side effects. The risk for contrast allergy is small because of the small molecular weight of microbubbles, and UCAs are applicable for patients with liver and renal dysfunctions because it is excreted by exhalation. ${ }^{6}$ The incidence of allergic events is comparable to magnetic resonance imaging (MRI) contrast agents. Life threatening hypersensitivity in abdominal applications have been reported with a rate of less than $0.002 \% .^{7,8}$ Previous allergic reaction to X-ray iodinated contrast agents does not necessitate the prophylactic use of antihistamines or steroids prior to injection of UCA because the two type of contrast agents are completely different. ${ }^{7}$ It is not necessary to perform renal function tests before administering UCAs, since they are not nephrotoxic. UCAs are not licensed on pregnant woman. Caution should be taken when using UCAs in patients with severe cardiopulmonary disease. ${ }^{8}$

\section{CE-EUS: TECHNICAL ASPECTS}

There are two main categories of CE-EUS; contrast-enhanced Doppler imaging and contrast-enhanced harmonic imaging. Doppler EUS allows characterization of target lesion on the basis of their vascularity. ${ }^{9}$ However, Doppler mode cannot depict very slowly flowing microcirculation and parenchymal perfusion. $^{9}$ A dedicated contrast-enhanced harmonic technology has become available for the enhancement of UCAs in EUS. This harmonic imaging technology is able to detect signals from the microbubbles in the microvasculature with very slow flow, without the burden of Doppler-related artifacts, and has enhanced the depiction and characterization of digestive diseases.
The image can be optimized by using a low mechanical index, which ensures minimal bubble disruption, and continuous realtime assessment of the microcirculation during the contrast uptake can be performed.

Microbubbles of UCAs oscillate (expand and contract) in the ultrasound field. The pattern and nature of the backscatter signal differs depending on the transmitted acoustic power. ${ }^{9}$ The power of the ultrasound is expressed as the mechanical index (MI). At low levels of acoustic power (low mechanical index $<0.1$ ), microbubbles demonstrate linear oscillation (reflected frequency equals impeding frequency). ${ }^{9}$ As the acoustic power increases, the bubbles produce nonlinear echoes from tissue, resulting in the generation of harmonic signals. ${ }^{9}$ At high acoustic powers (high mechanical index >0.6), destruction of the bubbles occurs, emanating a strong but transient signal. ${ }^{9}$ High mechanical index technology allow only intermittent scanning of the lesion during contrast enhancement. ${ }^{9}$ Conversely, the contrast specific harmonic mode operates under a low mechanical index, therefore, the UCAs are not destroyed, and the scanning fashion is continuous. ${ }^{10}$ These nondestructive technique require the administration of second-generation UCAs, which are able to reflect the ultrasound beam at a low mechanical index. The contrast-enhanced harmonic EUS was developed to allow the selective depiction of signals from microbubbles located in the microvasculature by filtering out signals from the tissue.

\section{IMAGING TECHNIQUES AND EVALUATION OF CE-EUS}

For SonoVue, which is the most commonly used UCA, the recommended dose is $2.4 \mathrm{~mL}$. The ultrasound processor was the Aloka Alpha 10 unit (Aloka, Tokyo, Japan), which incorporates 
dedicated software for the contrast harmonic imaging. The protocol consisted of EUS visualization of the pancreatobiliary area of interest and an injection of a $2.5 \mathrm{~mL}$ bolus of Sonovue followed by a $5 \mathrm{~mL}$ bolus of saline. This is best achieved by using a three-way stopcock.

\section{The steps in the procedure}

(1) A baseline investigation in B-mode is performed.

(2) After visualization of the target by EUS, the transducer is kept in a stable position while the imaging mode is shifted to a contrast harmonic imaging mode.

(3) UCAs must be prepared before use; vigorous shaking for some seconds creates the final solution that is ready for injection. The UCA is administered as a bolus injection followed by a 5 to $10 \mathrm{~mL}$ of additional saline flush. The needle diameter should not be smaller than 20-gauge to prevent loss of microbubbles due to mechanical impact during injection.

(4) Because of the dynamic nature of real-time CE-EUS, it is recommended that the investigation be recorded on video or digital media for at least 2 to 3 minutes.

(5) A single bolus is generally adequate, but further injections can be used if the examination after the first injection was inconclusive. The injection of SonoVue can be repeated after a 5-minute time interval.

\section{Image interpretation and evaluation}

\section{1) The timing of enhancement}

The arterial phase lasts approximately 30 to 45 seconds, during which the degree of enhancement increases progressively. ${ }^{11}$ The venous and late phase persists from 30 to 120 seconds, during which the degree of enhancement shows a plateau and then decreases progressively until signals have disappeared completely. ${ }^{11}$ The arterial phase provides information about the degree and pattern of vascularization. The venous and late phases provide information about the washout pattern of contrast from the lesion compared to surrounding tissue.

\section{2) The degree of enhancement}

When the target is a focal lesion within a parenchymal organ, the degree of enhancement needs to be compared to the surrounding parenchyma. On the basis of the degree of enhancement, the target lesion might present in a hyperenhancing, isoenhancing, hypoenhancing, or nonenhancing pattern. When the target has no tissue background with which to evaluate the degree of relative enhancement, it is important to describe the presence or absence of enhancement and its distribution (e.g., in septae within a cystic pancreatic lesion).

\section{3) Contrast distribution}

The distribution of enhancement should be clarified. When enhancement is seen, the enhancement can be described as homogeneous or heterogeneous.

\section{RECENT APPLICATIONS OF CE-EUS IN PANCREATOBILI- ARY DISEASES}

\section{Characterization of solid pancreatic masses}

Despite its efforts to implement criteria for the differential
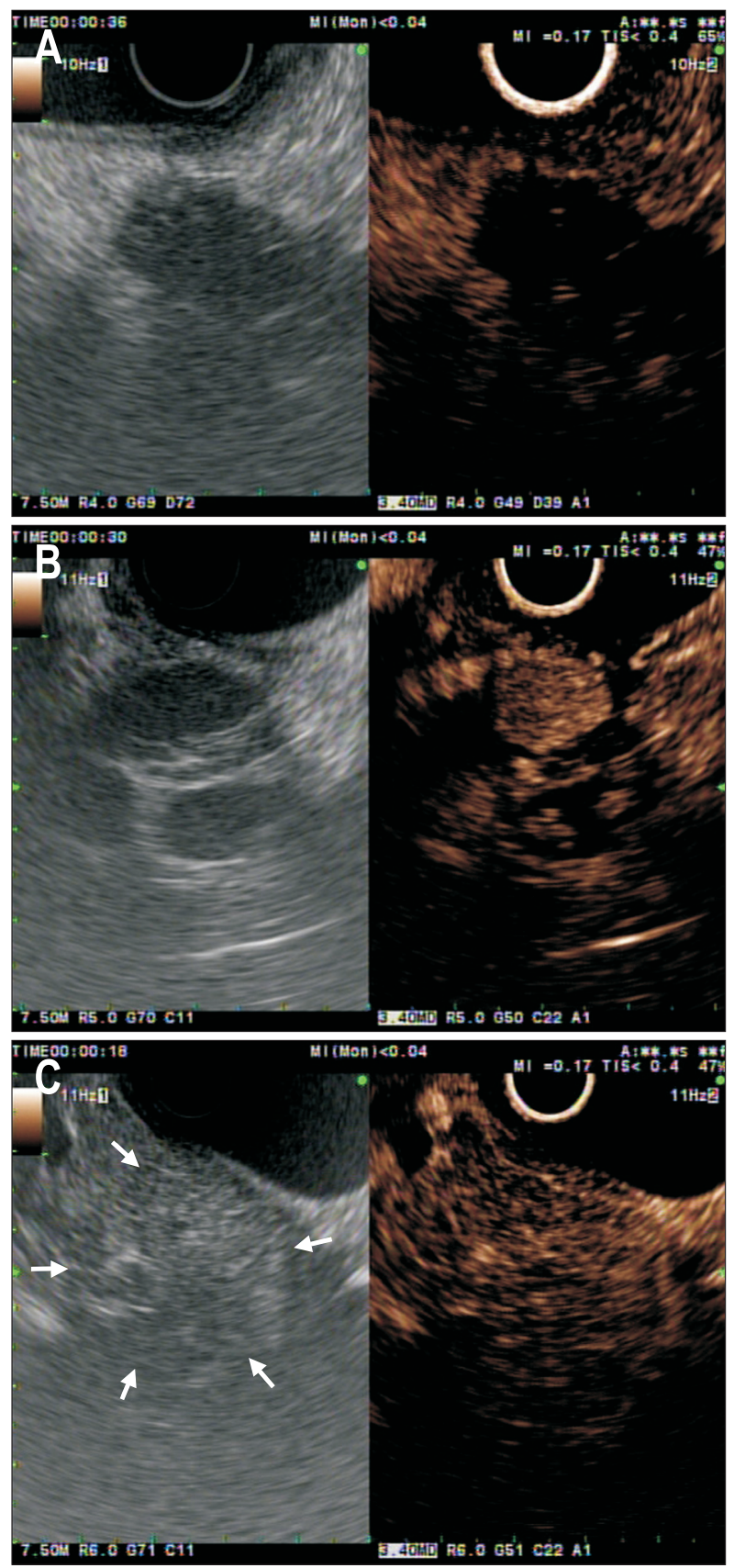

Fig. 1. Conventional endoscopic ultrasound (EUS; left panel) and contrast-enhanced EUS (CE-EUS; right panel) images of pancreatic tumors. (A) A pancreatic ductal adenocarcinoma with hypoenhancement. (B) A patient with pancreatic neuroendocrine tumor. The CEEUS image in the right panel shows a hypervascular pancreatic mass. (C) An inflammatory pseudotumor with isoenhancement. Conventional EUS (left panel) shows a tumor (white arrows) at the pancreatic head. The CE-EUS image (right panel) shows enhancement in this area similar to the surrounding parenchyma. 
diagnosis between pancreatic cancer and mass-forming chronic pancreatitis, the accuracy of EUS has never been higher than $75 \% .^{12}$ The image patterns of solid pancreatic masses were classified according to the density of vessels inside tumors compared with the surrounding pancreatic parenchyma as measured by CE-EUS. Pancreatic adenocarcinoma is typically hypoenhancing, presumably due to the desmoplastic reaction and low mean vascular density (Fig. 1A). ${ }^{13,14}$ Conversely, CE-EUS depicts most neuroendocrine tumors as a hyperenhancing pattern owing to their abundant arterial vascularization (Fig. 1B). ${ }^{15-17} \mathrm{Sev}$ eral studies have demonstrated that the majority of mass-forming focal pancreatitis exhibit an iso/hyperenhancement pattern (Fig. 1C). ${ }^{15,18-20}$ In the study by Gincul et al., ${ }^{21} 82 \%$ of the massforming focal pancreatitis showed an iso/hyperenhancement pattern. With the development of the techniques and the aid of clinical studies, CE-EUS has been assessed for its ability to differentiate solid pancreatic masses, with the sensitivity ranging from $80 \%$ to $96 \%$ and specificity ranging from $64 \%$ to $100 \%{ }^{22}$

CE-EUS may help localize a pancreatic mass that is not clearly visualized on EUS, and may help guide the target of biopsies within a lesion when performing fine-needle aspiration (FNA). ${ }^{9}$ When CE-EUS showed a hyperenhancement or isoenhancement pattern, it strongly supports benign EUS-FNA results. ${ }^{23}$ In contrast, a negative cytology result should not be considered a benign lesion in cases of hypoenhancement patterns, and repeated FNA are mandatory in these cases. The precise role of CE-EUS in the diagnosis of solid pancreatic mass needs to be further evaluated.

\section{Preoperative tumor staging}

Accurate preoperative T-staging, particularly regarding vascular involvement, is crucial in guiding the appropriate management for pancreatobiliary malignancies. Although EUS has been used widely in the diagnosis and T-staging of pancreatobiliary malignancies, the accuracy of EUS for vascular invasion appeared to be lower than has previously been reported. ${ }^{24}$ Tumor margin and the interface between tumor and vessel were more clearly demonstrated with contrast-enhancement. ${ }^{25}$ Imazu et al. ${ }^{25}$ compared the accuracy of EUS and CE-EUS for preoperative T-staging in 26 patients who underwent surgery. CE-EUS was significantly better than EUS alone for T-staging, with an overall accuracy of 92\% and 69\%, respectively. ${ }^{25}$ In addition, CE-EUS depicted the outline of the portal vein more clearly, which suggests that it is superior in diagnosing portal vein invasion by pancreatobiliary malignancies. ${ }^{25}$ The use of CE-EUS also contributed to the diagnosis of the depth of the cancer invasion. Enhancement of both the first and the third layers brought about enhanced contrast resolution between the gallbladder wall and the tumor, resulting in an accuracy in determining the T-staging. ${ }^{26}$

\section{Monitoring of local ablative treatment}

Recent research has focused on the promise of EUS-guided local ablation for treating pancreatic tumor. Diagnostic imaging in patients undergoing local ablation includes contrastenhanced computed tomography (CT), MRI, and/or EUS during pretreatment work-up and at certain intervals within the followup. Conventional EUS does not provide any reliable information about the efficacy of ablative treatment. The assessment of vascularization and altered perfusion is crucial to differentiate necrotic area from residual viable tumor. When EUS is used as the imaging modality for guiding ablations, the addition of UCAs can provide useful information. ${ }^{27,28}$ Pretreatment assessment of tumor vascularity in order to compare pre- and postablation patterns at the end of ablation treatment is mandatory. The typical imaging that indicates complete ablation is the disappearance of any previously visualized intralesional enhancement on CE-EUS. ${ }^{27,28}$ The size of the nonperfused volume of the necrosis achieved could be compared with the size of the pretreatment volume of the tumor. CE-EUS facilitates posttreatment followup because it improves visualization of altered pancreatic perfusion in the focal areas of pancreatic necrosis after ablation. ${ }^{27,28}$

\section{Pancreas cystic tumors}

The knowledge of the malignant potential of pancreas cystic tumors has a great impact on the decision to refer the patient for pancreatic surgery and leaves the physician with the question. ${ }^{29}$ The use of CE-EUS has recently been adopted in the assessment of pancreas cystic tumors. Ohno et al. ${ }^{30}$ reported that the type of mural nodules obtained by CE-EUS may be the reliable diagnostic tool between malignant and benign intraductal papillary mucinous neoplasm (IPMN). CE-EUS discriminates mural nodules from mucous clots in IPMNs by assessing the vascularity of the protrusions: mural nodules are vascular, whereas mucous clots are not. ${ }^{30}$ Serous cystadenoma is a benign cystic neoplasm, typically with a lobulated microcystic architecture with thin septae and wall, which are hyperenhanced on CE-EUS. ${ }^{18}$

\section{Gallbladder and biliary tract diseases}

Sometimes, motionless sludge ball in the gallbladder mimics gallbladder polyp or cancer on EUS. ${ }^{31}$ CE-EUS can easily depict biliary sludge as avascular lesions in the gallbladder (Fig. 2A) and the biliary tract, whereas it depicts apparent vessels in a gallbladder polyp and carcinoma. ${ }^{32}$ A differential diagnosis between adenomas and cholesterol polyps is important because adenomas have the potential to turn into cancer over time. Park et $a l^{33}$ identified that the majority of the adenomas and cholesterol polyps showed homogeneous and heterogeneous enhancement pattern, respectively (Fig. 2B). In their study, the sensitivity and specificity of CE-EUS for differentiating adenoma from cholesterol polyps were only 75\% and 67\%, respectively. ${ }^{33}$ The authors suggested that the quantitative analysis of CE-EUS may 


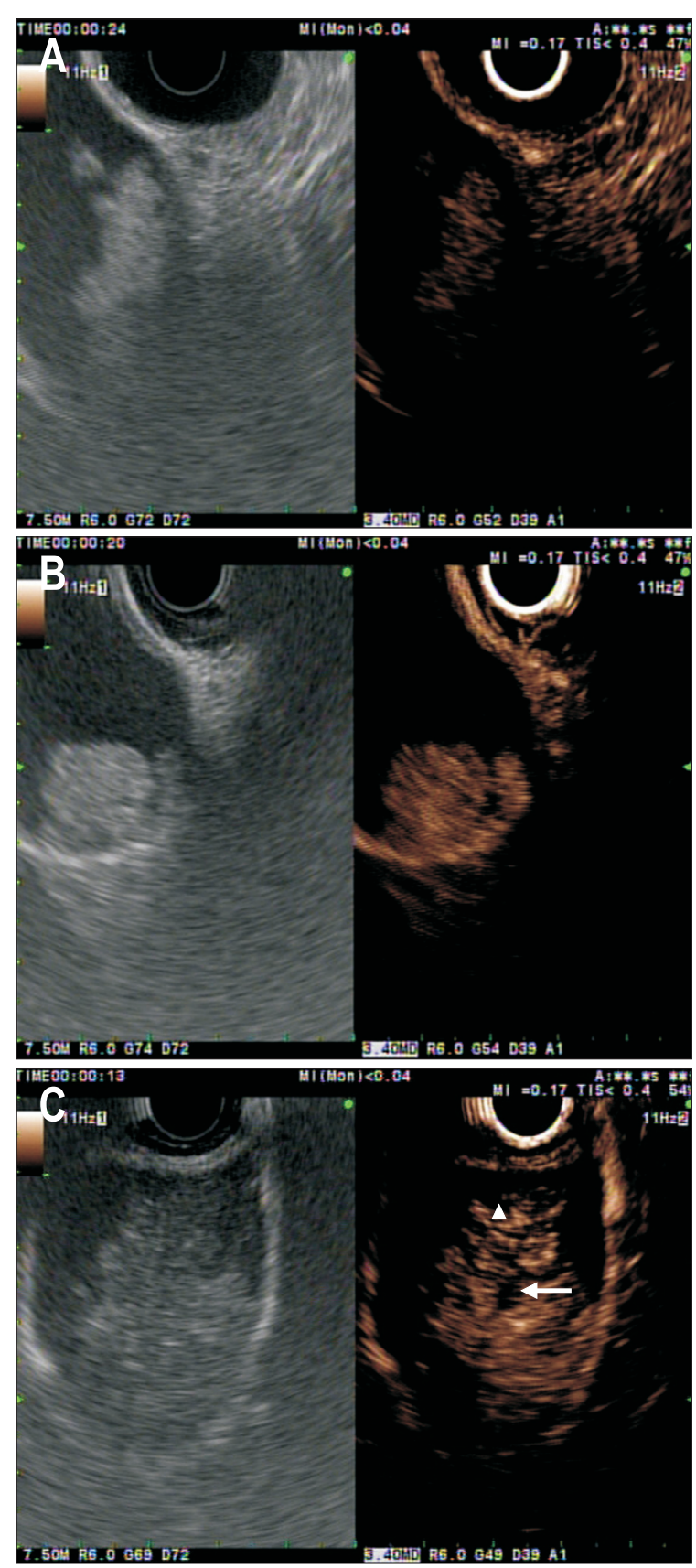

Fig. 2. Conventional endoscopic ultrasound (EUS; left panel) and contrast-enhanced EUS (CE-EUS; right panel) images of gallbladder polyoid lesions. (A) Conventional EUS (left panel) shows a motionless isoechoic mass in the gallbladder. Contrast-enhanced image (right panel) indicates that this area contains an unenhanced lesion (in this case, a gallbladder sludge ball). (B) Contrast-enhanced image (right panel) shows a homogeneous enhancement with regular spotty vessel (in this case, a gallbladder adenoma). (C) A patient with a gallbladder cancer. CE-EUS (left panel) indicates this area has irregular vessels (white arrowhead) and perfusion defects (white arrow).

be necessary to improve the diagnostic accuracy of CE-EUS in this setting. ${ }^{33}$ Choi et al. ${ }^{32}$ investigated the value of CE-EUS in determining the malignant potential of gallbladder polyps larger than $10 \mathrm{~mm}$. The vascular distribution of the lesion may be helpful in differentiation, for benign lesions often show dotted vessel enhancement, whereas malignant lesions often show tortuous vessel enhancement (Fig. 2C). ${ }^{32}$ In this study, the sensitivity and specificity for CE-EUS in diagnosing malignant gallbladder polyps were 94\% and 93\%, respectively, when considering the presence of irregular tumor vessels and perfusion defects as predictors of malignancy. ${ }^{32}$ Imazu et al..$^{34}$ evaluated CE-EUS in the diagnosis of patients with gallbladder wall thickening. They identified that an inhomogeneous enhancement pattern on $\mathrm{CH}-$ EUS is strongly predictive of a malignancy. ${ }^{34}$ The application of CE-EUS in the biliary system is under investigation and needs further validation.

\section{Prediction of malignant lymph nodes}

Although EUS provides highly accurate image of lymph nodes, the differential diagnosis of pathologic lymph nodes remains a challenge. Current sonographic criteria for malignant lymph node (round, hypoechoic, diameter $>1 \mathrm{~cm}$, and distinct margin) are helpful in targeting lesions, but problems exist with low specificity and overlap of these features with benign lymph nodes. ${ }^{35}$ The use of CE-EUS has been reported to be beneficial in differentiating between malignant and benign lymph nodes. ${ }^{15,36}$ A perfusion defect and irregular network-like vessels were identified as a sign of malignant lymph nodes. ${ }^{15,36}$

\section{Visceral vascular diseases}

Color Doppler EUS helps to depict abnormal blood flow, along with its direction and velocity inside the artery. The addition of UCA injection can enhance the depiction of vascular flow and help to describe the vascular structure clearly. ${ }^{37}$ Paik et al. ${ }^{37}$ evaluated the usefulness of CE-EUS in diagnosing visceral vascular diseases and in the assessment of hemodynamic characteristics required for optimized patient management. The focal stenosis of visceral artery, which is not seen on color Doppler EUS, could be detected on CE-EUS. ${ }^{37}$ The authors concluded that CE-EUS provides a real-time, angiography-like depiction of the visceral vessels and enables the accurate diagnosis of visceral vascular disease without exposure to radiation (Fig. 3). ${ }^{37}$

\section{FUTURE PERSPECTIVE}

So far, the technique of CE-EUS has been studied for evaluation of vascularity. The diagnostic accuracy of CE-EUS in detecting malignancies could be further improved by using a microbubble contrast agent engineered to bind proteins differentially expressed in the neovasculature of cancer. ${ }^{3}$ Neovascularization, the process of new vascular growth from existing vascular networks or circulating endothelial stem cells, plays an important role in tumor growth. ${ }^{38}$ Molecular imaging of neovascular markers, such as vascular endothelial growth factor receptor type 2 (VEGFR2), which plays a key role in tumor neovascularization of many cancers, including pancreatic cancer, 

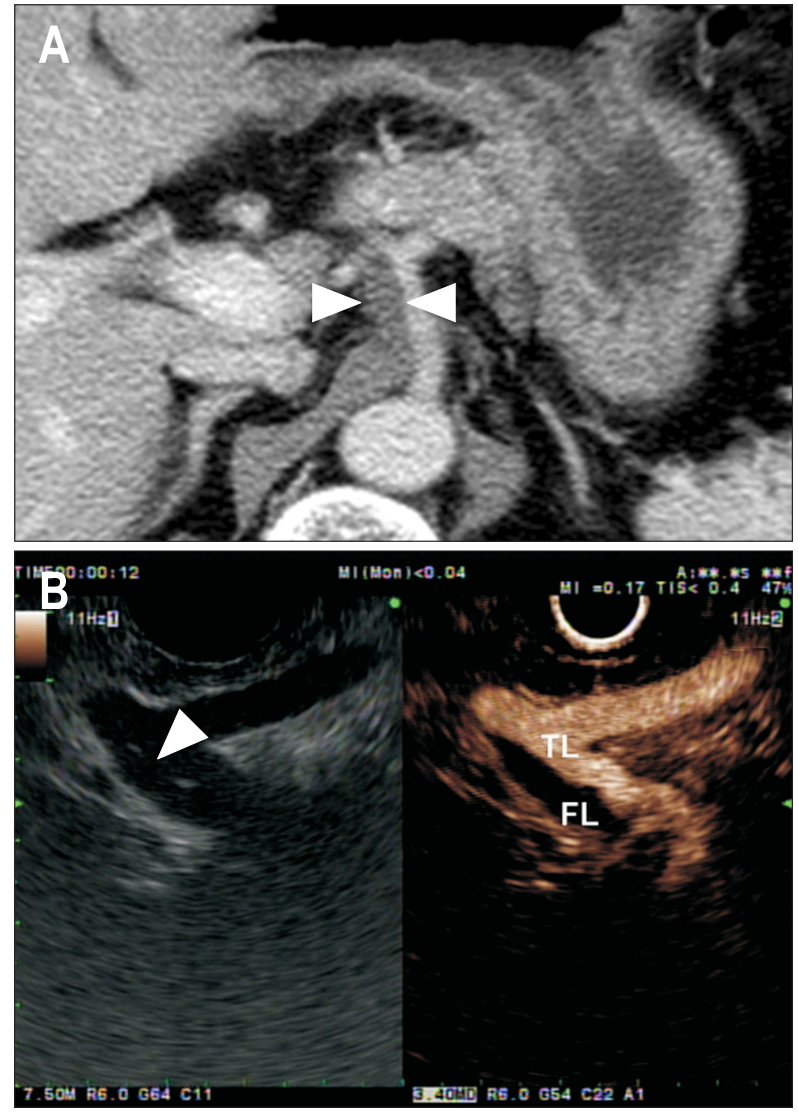

Fig. 3. Computed tomography (CT) and endoscopic ultrasound (EUS) findings in patient with celiac artery dissection. (A) Contrastenhanced CT scan shows periarterial soft tissue cuffing (white arrowheads) around the celiac artery. (B) EUS shows an intimal flap (white arrowhead) inside the artery and the true lumen (TL) and false lumen (FL).

may be a suitable approach for detecting pancreatic cancer at an early and treatable stage, just after the angiogenic switch has occurred during tumor progression. ${ }^{38,39}$ Recent study suggests that ultrasound with molecularly VEGFR2-targeted contrast agents allows the detection of early pancreatic cancer in highrisk patients. ${ }^{40}$ The addition of molecular imaging with microbubble UCAs targeted to angiogenic markers, such as VEGFR2, has great potential for enhancing earlier detection of pancreatic cancer. $^{40}$

\section{CONCLUSIONS}

The use of CE-EUS has proved beneficial in the evaluation of various pancreatobiliary diseases. The enhancing pattern gives the endoscopist a unique impression of the global microvasculature of the pancreas or biliary tract with a resolution. The main advantages of CE-EUS include real-time imaging of microvasculature and tissue perfusion, real-time guidance for intervention, and impressively good detail resolution. It is appropriate for repeated follow-up examinations due to the lack of radiation. It can be performed without any previous laboratory test for renal function as UCAs are not nephrotoxic. In addition, once the software is available, UCAs add relatively low costs to the basic EUS exam.

There is still a need for the more dedicated contrast agentspecific software and the objective assessment method. Further research is needed to exactly define the place of this technique in routine clinical practice and also to determine future indications.

\section{CONFLICTS OF INTEREST}

No potential conflict of interest relevant to this article was reported.

\section{REFERENCES}

1. Teshima CW, Sandha GS. Endoscopic ultrasound in the diagnosis and treatment of pancreatic disease. World J Gastroenterol 2014; 20:9976-9989.

2. Săftoiu A, Dietrich CF, Vilmann P. Contrast-enhanced harmonic endoscopic ultrasound. Endoscopy 2012;44:612-617.

3. Yip HC, Teoh AY, Chong CC, Lau JY. Current status and future applications of contrast-enhanced endoscopic ultrasonography. World J Gastrointest Endosc 2014;6:121-127.

4. Reddy NK, Ioncică AM, Săftoiu A, Vilmann P, Bhutani MS. Contrast-enhanced endoscopic ultrasonography. World J Gastroenterol 2011;17:42-48.

5. Sumi H, Hayashi D, Ohmiya N, et al. Contrast-enhanced endoscopic ultrasonography in digestive diseases. J Gastroenterol 2012; 47:1063-1072.

6. Toft KG, Hustvedt SO, Hals PA, et al. Disposition of perfluorobutane in rats after intravenous injection of Sonazoid. Ultrasound Med Biol 2006;32:107-114.

7. ter Haar G. Safety and bio-effects of ultrasound contrast agents. Med Biol Eng Comput 2009;47:893-900.

8. Piscaglia F, Bolondi L; Italian Society for Ultrasound in Medicine and Biology (SIUMB) Study Group on Ultrasound Contrast Agents. The safety of Sonovue in abdominal applications: retrospective analysis of 23188 investigations. Ultrasound Med Biol 2006;32:1369-1375.

9. Kitano M, Sakamoto H, Kudo M. Endoscopic ultrasound: contrast enhancement. Gastrointest Endosc Clin N Am 2012;22:349-358.

10. Dietrich CF, Ignee A, Frey H. Contrast-enhanced endoscopic ultrasound with low mechanical index: a new technique. Z Gastroenterol 2005;43:1219-1223.

11. Piscaglia F, Nolsøe C, Dietrich CF, et al. The EFSUMB Guidelines and Recommendations on the Clinical Practice of Contrast Enhanced Ultrasound (CEUS): update 2011 on non-hepatic applications. Ultraschall Med 2012;33:33-59.

12. Galasso D, Carnuccio A, Larghi A. Pancreatic cancer: diagnosis and endoscopic staging. Eur Rev Med Pharmacol Sci 2010;14:375- 
385.

13. Numata K, Ozawa Y, Kobayashi N, et al. Contrast-enhanced sonography of pancreatic carcinoma: correlations with pathological findings. J Gastroenterol 2005;40:631-640.

14. Lee TY, Cheon YK, Shim CS. Clinical role of contrast-enhanced harmonic endoscopic ultrasound in differentiating solid lesions of the pancreas: a single-center experience in Korea. Gut Liver 2013; 7:599-604

15. Kitano M, Sakamoto H, Matsui U, et al. A novel perfusion imaging technique of the pancreas: contrast-enhanced harmonic EUS (with video). Gastrointest Endosc 2008;67:141-150.

16. Fusaroli P, Eloubeidi MA. Diagnosis of pancreatic cancer by contrast-harmonic endoscopic ultrasound (EUS): complementary and not competitive with EUS-guided fine-needle aspiration. Endoscopy 2014;46:380-381.

17. Kitano M, Kudo M, Yamao K, et al. Characterization of small solid tumors in the pancreas: the value of contrast-enhanced harmonic endoscopic ultrasonography. Am J Gastroenterol 2012;107:303310.

18. Dietrich CF, Ignee A, Braden B, Barreiros AP, Ott M, Hocke M. Improved differentiation of pancreatic tumors using contrast-enhanced endoscopic ultrasound. Clin Gastroenterol Hepatol 2008;6: 590-597.e1.

19. Fusaroli P, Spada A, Mancino MG, Caletti G. Contrast harmonic echo-endoscopic ultrasound improves accuracy in diagnosis of solid pancreatic masses. Clin Gastroenterol Hepatol 2010;8:629634.e2.

20. Hocke M, Schulze E, Gottschalk P, Topalidis T, Dietrich CF. Contrast-enhanced endoscopic ultrasound in discrimination between focal pancreatitis and pancreatic cancer. World J Gastroenterol 2006;12:246-250.

21. Gincul R, Palazzo M, Pujol B, et al. Contrast-harmonic endoscopic ultrasound for the diagnosis of pancreatic adenocarcinoma: a prospective multicenter trial. Endoscopy 2014;46:373-379.

22. Gong TT, Hu DM, Zhu Q. Contrast-enhanced EUS for differential diagnosis of pancreatic mass lesions: a meta-analysis. Gastrointest Endosc 2012;76:301-309.

23. Deprez PH. Future directions in EUS-guided tissue acquisition. Gastrointest Endosc Clin N Am 2014;24:143-149.

24. Meining A, Dittler HJ, Wolf A, et al. You get what you expect? A critical appraisal of imaging methodology in endosonographic cancer staging. Gut 2002;50:599-603.

25. Imazu H, Uchiyama Y, Matsunaga K, et al. Contrast-enhanced harmonic EUS with novel ultrasonographic contrast (Sonazoid) in the preoperative T-staging for pancreaticobiliary malignancies. Scand J Gastroenterol 2010;45:732-738.

26. Hirooka Y, Goto H, Ito A, et al. Contrast-enhanced endoscopic ultrasonography in pancreatic diseases: a preliminary study. Am J Gastroenterol 1998;93:632-635.

27. Giday SA, Magno P, Gabrielson KL, et al. The utility of contrast- enhanced endoscopic ultrasound in monitoring ethanol-induced pancreatic tissue ablation: a pilot study in a porcine model. Endoscopy 2007;39:525-529.

28. Park do H, Choi JH, Oh D, et al. Endoscopic ultrasonographyguided ethanol ablation for small pancreatic neuroendocrine tumors: results of a pilot study. Clin Endosc 2015;48:158-164.

29. Oh HC, Seo DW, Song TJ, et al. Endoscopic ultrasonographyguided ethanol lavage with paclitaxel injection treats patients with pancreatic cysts. Gastroenterology 2011;140:172-179.

30. Ohno E, Hirooka Y, Itoh A, et al. Intraductal papillary mucinous neoplasms of the pancreas: differentiation of malignant and benign tumors by endoscopic ultrasound findings of mural nodules. Ann Surg 2009;249:628-634.

31. Choi WB, Lee SK, Kim MH, et al. A new strategy to predict the neoplastic polyps of the gallbladder based on a scoring system using EUS. Gastrointest Endosc 2000;52:372-379.

32. Choi JH, Seo DW, Choi JH, et al. Utility of contrast-enhanced harmonic EUS in the diagnosis of malignant gallbladder polyps (with videos). Gastrointest Endosc 2013;78:484-493.

33. Park $\mathrm{CH}$, Chung MJ, Oh TG, et al. Differential diagnosis between gallbladder adenomas and cholesterol polyps on contrast-enhanced harmonic endoscopic ultrasonography. Surg Endosc 2013; 27:1414-1421.

34. Imazu H, Mori N, Kanazawa K, et al. Contrast-enhanced harmonic endoscopic ultrasonography in the differential diagnosis of gallbladder wall thickening. Dig Dis Sci 2014;59:1909-1916.

35. Bhutani MS, Hawes RH, Hoffman BJ. A comparison of the accuracy of echo features during endoscopic ultrasound (EUS) and EUSguided fine-needle aspiration for diagnosis of malignant lymph node invasion. Gastrointest Endosc 1997;45:474-479.

36. Kanamori A, Hirooka Y, Itoh A, et al. Usefulness of contrast-enhanced endoscopic ultrasonography in the differentiation between malignant and benign lymphadenopathy. Am J Gastroenterol 2006;101:45-51.

37. Paik WH, Choi JH, Seo DW, et al. Clinical usefulness with the combination of color Doppler and contrast-enhanced harmonic EUS for the assessment of visceral vascular diseases. J Clin Gastroenterol 2014;48:845-850.

38. Hernot S, Klibanov AL. Microbubbles in ultrasound-triggered drug and gene delivery. Adv Drug Deliv Rev 2008;60:1153-1166.

39. Deshpande N, Ren Y, Foygel K, Rosenberg J, Willmann JK. Tumor angiogenic marker expression levels during tumor growth: longitudinal assessment with molecularly targeted microbubbles and US imaging. Radiology 2011;258:804-811.

40. Pysz MA, Machtaler SB, Seeley ES, et al. Vascular endothelial growth factor receptor type 2-targeted contrast-enhanced US of pancreatic cancer neovasculature in a genetically engineered mouse model: potential for earlier detection. Radiology 2015;274: 790-799. 